\title{
New insight into the pathogenesis of nail psoriasis and overview of treatment strategies
}

\author{
Alessandra Ventura' \\ Mauro Mazzeo' \\ Roberta Gaziano² \\ Marco Galluzzo' \\ Luca Bianchi' \\ Elena Campione' \\ 'Department of Dermatology, \\ University of Rome "Tor Vergata", \\ Rome, Italy; ${ }^{2}$ Department of \\ Experimental Medicine and Surgery, \\ Rome, Italy
}

\begin{abstract}
Psoriasis is a chronic inflammatory disease affecting up to $3 \%$ of the general population. The prevalence of nail involvement in psoriasis patients varies between $15 \%$ and $79 \%$. While the nails represent a small portion of the body surface area, psoriasis in these areas can have a disproportionate influence on a patient's physical and psychosocial activities. Differential diagnosis between an onychomycosis and a psoriatic nail could be challenging; nevertheless, coexistence of onychomycosis and nail psoriasis also occurs and both are common disorders in the general population. Nail psoriasis can be difficult to treat. Treatment of nail psoriasis should consider the body surface area of skin disease, psoriatic arthritis, severity of nail disease, and the impairment in the quality of life. All patients should be tested for onychomycosis before starting a therapy. This recommendation is underlined by the fact that nail psoriasis is mostly treated by immunosuppressive drugs, like steroids, methotrexate, or biologics, which may aggravate mycotic nail infections. Conventional systemic therapy, such as use of steroids, cyclosporine, methotrexate, and retinoid in the long term, can cause organ toxicities. Currently, use of apremilast and tofacitinib favors an early healing of nail psoriasis because they act directly on the pathogenic targets, distressing the inflammatory signals associated with the initiation and maintenance of the disease activity, and as with several conventional synthetic disease modifying antirheumatic drugs, they are characterized by the convenience of oral administration. The number of treatment options has increased considerably in recent years; however, given the heterogeneity of the disease, the therapy should be personalized to individual cases.
\end{abstract}

Keywords: nail, psoriasis, onychomycosis

\section{Introduction}

Psoriasis is a chronic inflammatory disease affecting up to $3 \%$ of the general population. The disease is characterized by epidermal hyper-proliferation resulting in erythematous-squamous skin plaques that may cover large body areas; in $\sim 30 \%$ of patients it is characterized by a seronegative spondyloarthritis. ${ }^{1}$

Psoriasis is considered a multiorgan disorder that requires a multidisciplinary approach and an appropriate management that takes into consideration a number of comorbidities. In fact, several studies revealed the association between psoriasis and a number of disease-related comorbidities including blood hypertension and cardiovascular diseases, obesity, type II diabetes, nonalcoholic fatty liver disease, anxiety, depression, and inflammatory bowel disease..$^{2-6}$

The disorder, in its entirety, is associated with a high degree of morbidity including a notable impact on social relationships, mental health, and work-related activities. ${ }^{6,7}$ The disease can have a substantial negative influence on a patient's quality of life (QOL), especially psoriasis of vastly visible areas of the body including face, hands, scalp, and nails, and it is associated with physical impairment and pain. Psoriasis
Correspondence: Alessandra Ventura Department of Dermatology, University of Rome "Tor Vergata" 8I Oxford Street, 00133 Rome, Italy Email alessandra.ventura1987@gmail.com
Drug Design, Development and Therapy 2017:II 2527-2535

(c) (1) (8) ๑ 2017 Ventura et al. This work is published and licensed by Dove Medical Press Limited. The full terms of this license are avalable at https:/www.dovepress.com/terms.php cc) hereby accept the Terms. Non-commercial uses of the work are permitted without any further permission from Dove Medical Press Limited, provided the work is properly attributed. For permision for commercial use of this work, please see paragraphs 4.2 and 5 of our Terms (https://www.doveppess. con/terms.php). 
affects a patient's QOL sometimes more than other chronic diseases such as diabetes, cardiovascular disease, and rheumatoid arthritis. ${ }^{5,8-10}$

While the most specific features of psoriasis are skin manifestations, nails are commonly involved. ${ }^{11}$ The prevalence of nail involvement in psoriasis patients varies between $15 \%$ and $79 \%{ }^{11-13}$ It is uncommon in children, and the prevalence ranges from $7 \%$ to $13 \%$, whereas in adult patients, nail psoriasis is common - in the absence of skin and joint diseases - and $5 \%-10 \%$ of adult patients are reported to be affected. ${ }^{13}$

Approximately $90 \%$ of psoriatic patients develop nail psoriasis during their lifetimes, and it is not related to gender or age. ${ }^{14,15}$ McGonagle et al described that nail, scalp, and intergluteal skin involvement are unfavorable signs and are often predictors of Psoriatic Arthritis (PsA) evolution. ${ }^{16}$ Nail involvement is frequently observed in association with psoriatic arthritis, and several studies have described an incidence of $80 \% .{ }^{14-18}$ Nail psoriasis is often related to a protracted duration of psoriasis and severity of skin and joint involvement. ${ }^{19}$ Furthermore, psoriatic nail disease may be considered as a risk factor for the development of psoriatic arthritis. ${ }^{20}$

\section{Clinical features of nail psoriasis}

Nardo Zaias, in 1969, firstly described the pathophysiology of nail psoriasis. ${ }^{21}$ The nail bed, nail matrix, hyponychium, and nail folds can be affected by nail psoriasis. The most observed forms are psoriasis of the nail matrix, nail bed, and nail fold. ${ }^{12}$ Pitting, leukonychia, red spots of the lunula, transverse grooves (Beau's lines), and crumbling of the nail plates are the typical signs of psoriasis of the nail matrix. ${ }^{21}$ Oil-drop discoloration, splinter hemorrhages involving the distal third of the nail plate, subungual hyperkeratosis, and/or detachment of the nail plate from the nail bed (onycholysis) are the characteristic marks of the nail bed involvement. ${ }^{12}$ Psoriasis of the periungual region is characterized by paronychia. ${ }^{12}$

The severity of nail psoriasis is evaluated by Nail Psoriasis Severity Index (NAPSI) which is a numeric, reproducible, objective, and simple tool. According to this index, each nail is divided into four quadrants, each of which is assessed for the presence of any signs of psoriasis in the nail matrix such as pitting, leukonychia, red spots in the lunula, nail plate crumbling and nail bed as oil-drop discoloration, onycholysis, hyperkeratosis, and splinter hemorrhages. ${ }^{22}$ This scale is used to evaluate the severity of nail bed psoriasis and nail matrix psoriasis based on the area of involvement in the nail unit. NAPSI is useful during clinical trials for assessing response to treatment of patients with psoriatic nails. ${ }^{22}$
Different clinical presentations are associated with nail psoriasis according to the nail structure apparatus. All the pathognomonic signs of nail psoriasis are not exclusive and may be found in several other nail disorders. Differential diagnosis between an onychomycosis and a psoriatic nail could be challenging; nevertheless, coexistence of onychomycosis and nail psoriasis also occurs and both are common disorders in the general population. ${ }^{23}$

Klaassen et al reported in their study a higher prevalence of onychomycosis in patients affected by psoriasis of the nail compared to the non-affected population. ${ }^{18}$ Several studies hypothesized that morphological defects in psoriatic nails are predisposing factors for onychomycosis and that onychomycosis could act as a Koebner phenomenon for the development of the psoriatic nails. ${ }^{24}$ In healthy nails, the nail plate acts as a natural barrier counteracting the development of fungal infections, whereas in psoriatic patients, the defective nail plate may be predisposed to fungal infection. ${ }^{25}$ Several studies have shown that patients with the highest NAPSI scores were most likely to test positive for fungal colonization. ${ }^{26,27}$

\section{Pathogenesis}

Psoriasis seems to be a multifactorial disorder whose rigorous underlying mechanism is still uncertain, and environmental factors, genetic susceptibility, abnormal function of keratinocytes, and dysregulation of innate and acquired immune response are all assumed. ${ }^{28,29}$

Certain infections, such as bacterial and fungal infections, especially Candida albicans have been shown to be involved and to play a role in the exacerbation and maintenance of the disease. in fact, psoriasis is a systemic inflammatory disease in which dysregulation of the immune system results in overexpression of inflammatory cytokines. Some of these cytokines are involved in host defense against common infections, including Candida. ${ }^{30-33}$ Candida can stimulate the production of superantigens, determining nonspecific T-cell activation and secretion of cytokines that can initiate the psoriatic process. ${ }^{34,35}$ Consequently, candidiasis is a documented trigger for psoriasis exacerbations and persistence. Candida could act as a trigger for the exacerbation of skin and nail psoriasis through the same LL-37 (cathelicidin) pathway; in particular, in the psoriatic nail, Candida could activate the antimicrobial peptide, LL-37, produced by epithelial nail bed cells that induce interleukin (IL)-23 production by dendritic cells and macrophages, which consequentially activate Th17, determining the cytokine overflow theory and acting as a trigger for the exacerbation of nail psoriasis. ${ }^{30-36}$ 
Previous findings demonstrated an increased expression of tumor necrosis factor (TNF)- $\alpha$, nuclear factor-kappa B, IL-6, and IL-8 in psoriasis-affected nails, which is consistent with the findings of a study on lesional psoriatic skin. ${ }^{37}$

Rashmi et al described an imbalanced cytokine milieu in psoriatic lesions, with the presence of increased levels of TNF- $\alpha$, interferon- $\alpha$, IL-2, IL-6, IL-8, IL-12, and leukemic inhibitory factor-1 and reduced levels of IL-1, IL-4, IL-5, and IL-10. ${ }^{38}$ The critical role of IL-23/Th17 axis at tissue level is indicated by the increased levels of IL-23, IL-23R and Th17 cytokines revealed in psoriatic skin, especially in lesional versus non-lesional skin. ${ }^{39}$

IL-10 is an anti-inflammatory and immunosuppressive cytokine that plays an important role in the regulation of the immune response. Several studies demonstrated a downregulation of IL-10 in psoriatic skin lesions. ${ }^{40}$ In contrast, Saulite et al found an increased expression of IL-10 in the affected nail bed suggesting unique pathways of psoriatic nail disease and the nail as an immune-privileged site. ${ }^{41-43}$ However, those results suggest that although the milieu of some of the distinctive inflammatory cytokines and chemokines appears to be consistent with that described in psoriatic skin lesions, there seems to be some distinctive peculiarities for nail psoriasis, thereby confirming the hypothesis that nails act as immune-privileged sites. Different therapeutic responses to monotherapy are due to the distinctive anatomic characteristics of the nail (Figure 1). ${ }^{41}$

\section{Therapeutic strategies}

There are limited therapeutic strategies for the management of nail psoriasis. Most conventional treatments have been used for nail psoriasis, but their efficacies are limited and can cause side effects; relapses are common and any noticeable nail improvement will take a long time..$^{37,44-46}$

Therapeutic management is based on clinical presentation, as well as patient-related factors. Most patients have mild nail psoriasis without arthropathic disease or severe skin psoriasis. ${ }^{28}$ These patients may be suggested topical therapy, while systemic therapy is suggested in patients affected by severe nail psoriasis and in those with major impact on QOL

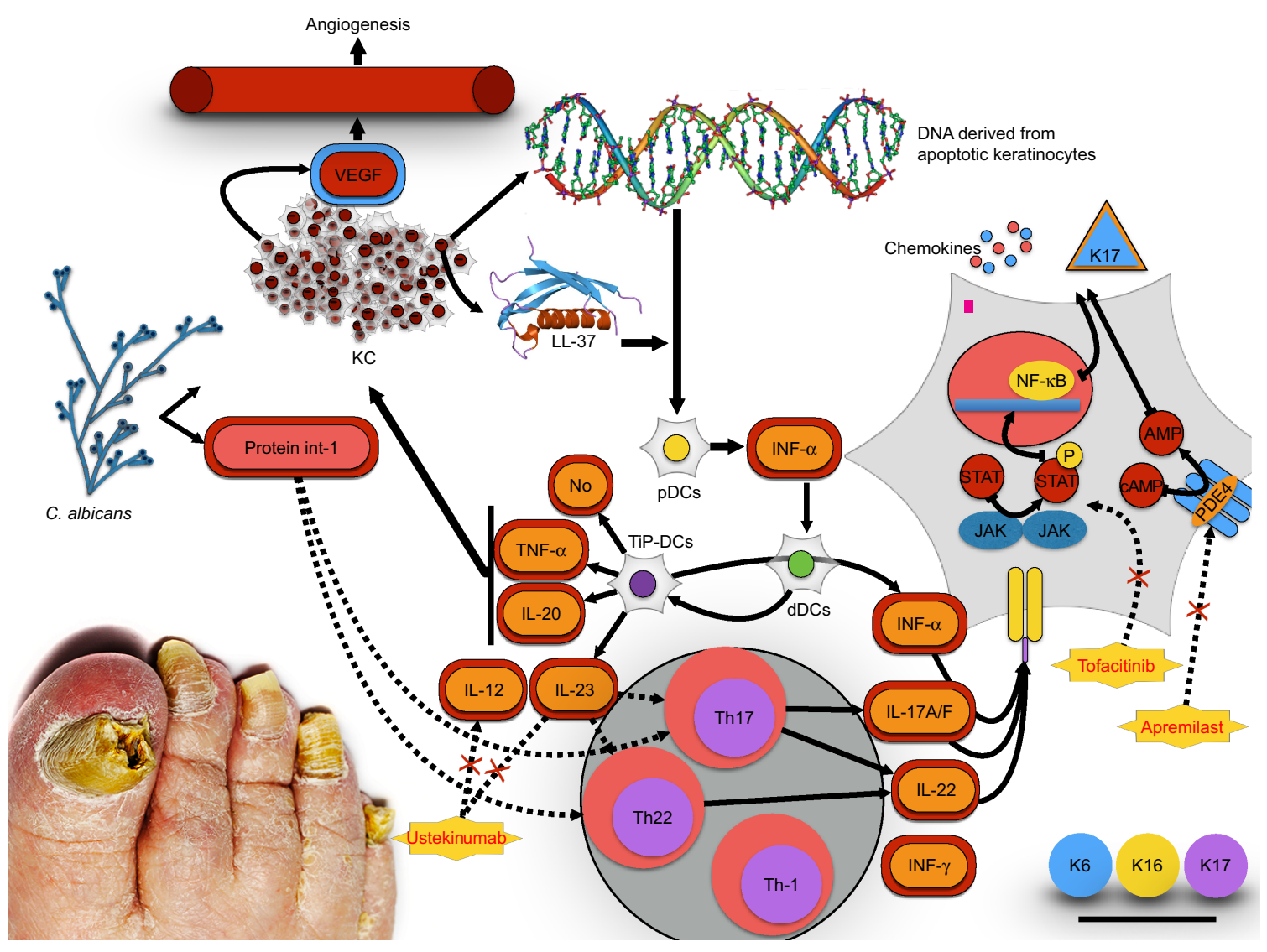

Figure I The cytokines axis in psoriasis. IL-23-, IL-I7-axis-related mediators are overexpressed in lesional psoriatic skin and nails. TNF $\alpha$-/iNOS-producing dendritic cells (TiP-DCs) are activated by various cells and stimuli including Candida albicans. Candida activates ThI7 and Th22 to produce IL-22 and IL-I7A/F. Keratinocytes are the keyresponding cells to this pathway.

Abbreviations: VEGF, vascular endothelial growth factor; IL, interleukin; TNF, tumor necrosis factor; iNOS, inducible nitric oxide synthase; KC, keratinocytes. 
or with moderate-to-severe psoriatic skin lesions or PsA. Age, burden of disease experienced, comorbidities, individual patient medication preferences, and risks of treatment should be considered for the treatment strategy.

Topical treatments are steroids, vitamin D3 analog calcipotriol or tacalcitol or calcitriol used in monotherapy or in combination with corticosteroids, tazarotene, topical calcineurin inhibitors, 5-fluorouracil. Vitamin D analogs normalize epidermal cell proliferation and differentiation, as well as production and release of proinflammatory cytokines. $1,25(\mathrm{OH}) \mathrm{D}$ has been shown to have an antiproliferative effect on keratinocytes. ${ }^{47}$ In particular, low concentration of vitamin D stimulates keratinocyte proliferation in vitro, while at higher pharmacological doses, a clear inhibitory influence has become evident. ${ }^{48}$

Furthermore, vitamin D acts as an immunomodulator inhibiting the proliferation of $\mathrm{T}$ lymphocytes and stimulates the generation of $\mathrm{CD}^{2} 5^{+} / \mathrm{CD} 4^{+}$Tregs, a phenotype of T cells endorsing tolerance and inhibiting immunity after stimulation with antigen. ${ }^{48}$ Additionally, vitamin D stimulates the expression of $\mathrm{C}-\mathrm{C}$ chemokine receptor type 10 on the surface of $\mathrm{T}$ lymphocytes, which is involved in T-cell mediated skin inflammation, determining $\mathrm{T}$ lymphocytes migration from dermal blood vessels to epidermal keratinocytes. ${ }^{48}$ In conclusion, vitamin D aids to defend from opportunistic infections, inducing autophagy and supporting the innate skin barrier, thereby stimulating endogenous antimicrobial peptides expression. ${ }^{48}$

Recent studies have shown that $1,25(\mathrm{OH}) \mathrm{D}$ values are significantly lower in psoriatic patients than in control healthy subjects. ${ }^{47}$ Vitamin D3 derivatives appear to be more successful in treating nail bed psoriasis than nail matrix. ${ }^{47}$ Tazarotene is a retinoid that has been shown to have antiproliferative, differentiation normalizing, and anti-inflammatory effects, and it is also approved for the treatment of psoriasis. ${ }^{48-53}$ Topical calcineurin inhibitors such as cyclosporine and tacrolimus are effective in treating both nail matrix signs of nail psoriasis (pitting) and nail bed signs (hyperkeratosis, onycholysis, crumbling, and oil-drop discoloration). No local or systemic side effects were reported during the clinical trial except for a yellowish discoloration after long-term application of cyclosporine.

Topical therapy for nail psoriasis has the evident advantage of treating the nail apparatus without exposing the rest of the organs to the risk of adverse events; however, in patients affected by severe skin psoriasis or PsA, systemic treatments offer a valuable alternative. Conventional systemic therapy, such as use of cyclosporine, methotrexate, and retinoid in the long term, can cause organ toxicities. ${ }^{54}$
Methotrexate has been shown to improve NAPSI score in several studies; however, its use is limited by a wide range of potential side effects, including hepatotoxicity, ulcerative stomatitis, lymphopenia, nausea, and low white blood cell count. ${ }^{55-58}$ The calcineurin inhibitor cyclosporine is an immunosuppressive drug characterized by reasonable efficacy in the treatment of nail bed and nail matrix signs of psoriasis. Unfortunately, serious adverse reactions such as renal dysfunction, hypertension, fatigue, headache, paresthesia, hypertrichosis, gingival hyperplasia, and gastrointestinal disorders have been reported. Furthermore, the prolonged use of cyclosporine may play a role in the development of renal failure and several malignancies. ${ }^{59,60}$

Acitretin, which belongs to the retinoid family, regulates cellular differentiation and also possesses anti-inflammatory properties. Acitretin has been shown to have a moderate efficacy particularly on nail signs of psoriasis, although its efficacy is limited by common side effects including cheilitis, dry mouth, and skin exfoliation. ${ }^{61,62}$ New molecules have been studied to improve the QOL of psoriasis patients focusing on the new discoveries. Psoriasis is characterized by anomalous immune response and determined by self-cytokine networks. ${ }^{63}$ The importance of these adverse effects has stimulated the development of new therapies characterized by high affinity and a safety profile.

Historically, anti-TNF- $\alpha$ biological agents such as infliximab, adalimumab, golimumab, certolizumab as well as etanercept, a recombinant TNF- $\alpha$ decoy receptor, have been used to treat psoriasis. TNF- $\alpha$ is a proinflammatory cytokine that plays a major role in psoriasis stimulating keratinocyte proliferation, inflammatory infiltrate in the epidermis and preventing keratinocyte apoptosis. The Anti-TNF- $\alpha$ family has been demonstrated to be a reasonably safe and effective treatment for plaque-type psoriasis, PsA, and nail psoriasis. Infections, such as mycobacterium tuberculosis, demyelinating diseases, congestive heart failure, induction of the formation of autologous antibodies, and production of antibodies neutralizing anti-TNF- $\alpha$ drugs were described as adverse reactions related to the anti-TNF- $\alpha$ agent. ${ }^{64}$

Infliximab, a chimeric anti-TNF- $\alpha$ immunoglobulin (Ig) G1 monoclonal antibody that consists of human antibody constant regions and murine variable regions, was approved for PsA and plaque-type psoriasis in 2005/2006. Several studies have shown the valuable effect of infliximab on nail psoriasis - both nail bed and nail matrix psoriasis. Also in patients with severe nail psoriasis at baseline, an excellent degree of response can be achieved after 22 weeks of therapy. ${ }^{65}$ The data indicate that infliximab is effective for 
psoriatic nail disease in the context of severe skin and/or joint involvement.

Adalimumab is a fully human anti-TNF- $\alpha$ IgG1 monoclonal antibody. Several studies have emphasized adalimumab to be an effective choice of treatment for psoriasis of the nail bed and nail plate. A prospective study reported a decreased incidence of onychomycosis in patients treated with adalimumab for 24 weeks. Significant improvement was noted as early as week 12 with regard to both fingernails and toenails in patients treated with adalimumab. ${ }^{66}$

Etanercept is a fusion protein of the TNF- $\alpha$ receptor and $\mathrm{Fc}$ end of the IgG1 antibody. Barrera et al reported an average reduction of $51 \%$ in the NAPSI score in 562 patients enrolled in this study after 54 weeks of therapy. ${ }^{67}$ The efficacy of etanercept on nail psoriasis was evident after 24 weeks of treatment, with greater improvements observed after 48 weeks. ${ }^{68,69}$ In conclusion, treatment of nail psoriasis with etanercept should be considered in the context of treating moderate-to-severe psoriasis with the aim of achieving improvement of both skin and psoriasis nail lesions.

Golimumab is a human monoclonal anti-TNF- $\alpha$ antibody. The randomized controlled trial, GO-REVEAL study, showed a mean NAPSI improvement of $52 \%$ at 52 weeks. ${ }^{70}$ Substantial improvements in nail symptoms, evaluated by the NAPSI and physician's global assessment of psoriatic nail disease, were observed in golimumab-treated patients as early as 24 weeks and were preserved or improved through 52 weeks. ${ }^{70}$ Golimumab should be considered in patients with nail and joint involvement as an advantageous treatment strategy.

Certolizumab pegol is a humanized mouse monoclonal antibody to TNF- $\alpha$ that is chemically modified by PEGylation, and consequentially it has a prolonged half-life in patients. Certolizumab confirmed the NAPSI improvement of the other anti-TNF drugs, the clinical trial showed that the NAPSI change from baseline at week 24 was $-52 \%$ with certolizumab pegol $200 \mathrm{mg}$ every 2 weeks and $-59 \%$ with certolizumab pegol $400 \mathrm{mg}$ every 4 weeks versus $-32 \%$ with placebo. ${ }^{71}$ Certolizumab pegol should be considered in patients with nail and joint involvement. Advances in understanding the pathogenesis of the disease have led to the development of new treatment strategies. ${ }^{72,73}$ Immunological research has recently pointed out the central role of IL-17 and IL-23 in the pathogenic pathway of psoriasis.

Ustekinumab is a human anti-IL-12/23 IgG1 monoclonal antibody. Rich et al described a mean NAPSI improvement of $46.5 \%$ within 24 weeks both in nail bed and nail matrix psoriasis. ${ }^{74}$ PHOENIX-1 study was a placebo-controlled study carried out in 766 psoriasis patients treated with ustekinumab
$45 \mathrm{mg}$, of whom $70 \%$ were affected by nail psoriasis. Nail improvement was higher in patients with a good Psoriasis Area Severity Index (PASI) response and the improvement in the NAPSI scale ranged from $30 \%$ to $57 \%$ at 24 weeks. ${ }^{74}$

Several other studies have been performed that have confirmed these results. ${ }^{75,76}$ Studies on ustekinumab demonstrated nail responses similar to those obtained with other biologics (about 24 weeks of treatments). Contraindications and adverse events of ustekinumab are similar to anti-TNF $\alpha$ treatments. $^{77}$

IL-17, firstly described by Yao et al in $1995,{ }^{78}$ is a family of proinflammatory cytokines that consists of IL-17A, IL-17B, IL-17C, IL-17D, IL-17E, and IL-17F, secreted by T cells, natural killer cells, mast cells, and neutrophils. ${ }^{78}$ IL-17A is produced predominantly by $\mathrm{Th} 17$, a subset of $\mathrm{CD}^{+} \mathrm{T}$ cells. Anomalous production of IL-17A is strongly implicated in the pathogenesis of psoriasis and other autoimmune diseases such as rheumatoid arthritis, chronic noninfectious uveitis, and Crohn's disease. Its neutralization has potential utility in other autoimmune disorders. ${ }^{79,80}$

Based on the evidence available, regarding the role of Th17 cells in psoriasis and psoriatic arthritis, three therapeutic agents against IL-17A have been developed: secukinumab, ixekizumab, and brodalumab. Significant reductions in NAPSI scores were observed using ixekizumab as early as 2 weeks, through 20 weeks, and in an openlabel extension. ${ }^{81,82}$ Complete remission of nail disease was achieved in a high proportion of patients: $43 \%$ at week 44 and $51 \%$ at week $68 .{ }^{82}$ Although few data are available, due to the recent approval of the drug, ixekizumab should be considered in patients affected by severe skin psoriasis.

Apremilast is a selective inhibitor of phosphodiesterase 4 that breaks down cyclic adenosine monophosphate (cAMP). An increase in cAMP levels downregulates the expressions of TNF $\alpha$, IL-17, IL-23, and many others, and upregulates the anti-inflammatory IL- $10 .{ }^{83}$

A $50 \%$ reduction in NAPSI was achieved for $33.3 \%$ and $14.9 \%$ of patients given apremilast $(30 \mathrm{mg}$ ) twice a day and placebo, respectively, at 16 weeks. At week 32 (an openlabel study), a 50\% reduction in NAPSI score response was observed in $45.2 \%$ of patients treated with apremilast by Papp et al in a Phase III, multicenter, double-blind, placebocontrolled study. ${ }^{83}$

ESTEEM 1 and ESTEEM 2 evidenced a target NAPSI improvement of $43 \%$ and $60 \%$, respectively, after 32 weeks of therapy, which was maintained through week $52 .{ }^{84,85}$ Apremilast has been shown to be efficient in the treatment of both nail matrix and nail bed psoriasis (Figure 2). Furthermore, 
Week 0

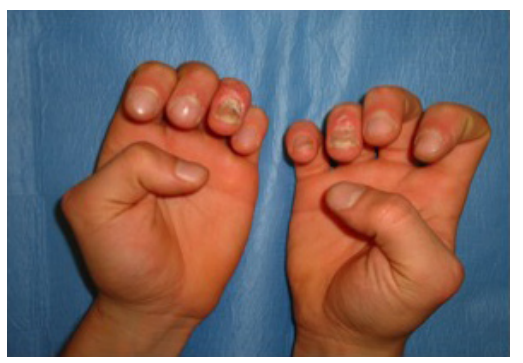

Week 12

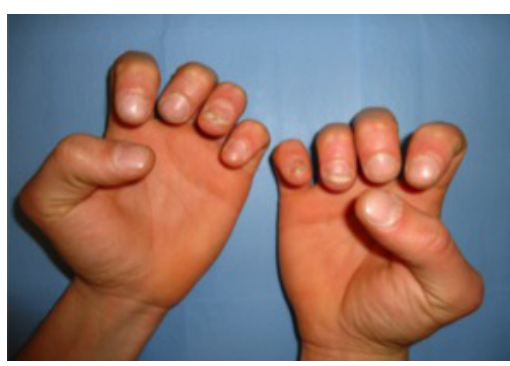

Week 24

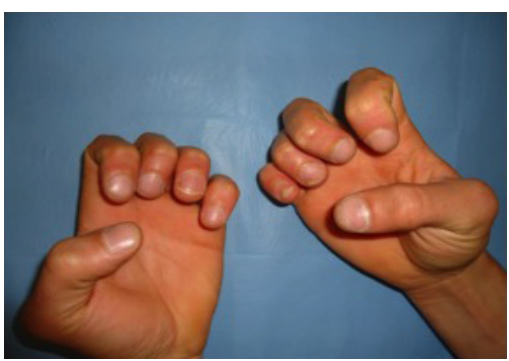

Figure 2 Patient affected by nail psoriasis at baseline (week 0). The same patient after 12 weeks and 24 weeks of treatment with apremilast.

apremilast was generally well tolerated during Phase III clinical trials, and in particular, most adverse events were mild or moderate in intensity and the incidence rate did not appear to increase over time. ${ }^{44}$ The convenience of oral administration of apremilast is associated with its safety profile, and the tolerability of the side effect profile makes this medication an attractive choice for clinicians. ${ }^{83,84}$ Apremilast should be considered in patients with joint and skin involvement. Apremilast is not only effective for moderate-to-severe psoriasis or PsA but it is also characterized by a good safety profile and does not require laboratory monitoring as with traditional biological agents.

Tofacitinib is an oral Janus kinase inhibitor that recently has been investigated for psoriasis. Papp et al during Phase III studies described significant dose-dependent improvements in the NAPSI score with tofacitinib treatment in patients affected by nail psoriasis, suggesting that the JAK pathway is a critical therapeutic target of the disease ${ }^{86}$ It also offers the convenience of oral administration. In conclusion, oral tofacitinib is an effective, well-tolerated treatment option for moderate-to-severe chronic plaque psoriasis.

\section{Discussion}

While the nails represent a small portion of the body surface area, psoriasis in this area can have a disproportionate influence on a patient's physical and psychosocial activities. ${ }^{6}$ This review highlighted the primary importance of the nail involvement in the psoriatic disease. Furthermore, we emphasize the role of Candida in inducing and maintaining inflammation through IL-17; moreover, keratinocytes express IL-17 receptor and are involved in the production of aberrant K17. ${ }^{35,41}$

Therefore, we suggest ruling out onychomycosis in patients with nail abnormalities before concluding that the patients are affected by nail psoriasis and, in psoriatic patients, excluding the comorbidities with a fungal infection before starting treatment. This recommendation is underlined by the fact that nail psoriasis is mostly treated with immunosuppressive drugs, like steroids, methotrexate, or biologics, which may aggravate mycotic nail infections. ${ }^{44}$ To manage a patient co-affected by onychomycosis and psoriasis, we suggest to initially treat the fungal infection for at least 3 months using a systemic antifungal therapy and to avoid local topical steroids and subsequently treat the psoriatic disease..$^{24,62}$

The commonly used disease modifying antirheumatic drugs (DMARDs) can cause adverse effects that require monitoring, ${ }^{54}$ while anti-TNF- $\alpha$ (eg, adalimumab, etanercept, infliximab, certolizumab pegol, and golimumab) can cause infections, malignancies, and production of antidrug antibodies that can impact effectiveness; administration of TNF- $\alpha$ is through injection or infusion. ${ }^{64,66,70,71}$

Currently, two recent meta-analyses - regarding adalimumab, etanercept, golimumab, and infliximab - showed that differences in terms of efficacy of these drugs cannot be demonstrated. ${ }^{87,88}$ Additionally, in one of these studies, no significant differences in terms of safety of these drugs were established..$^{87}$ Though the mechanism of action of the anti-TNF biologic drugs is similar, some molecular differentiations might justify the successful switch from one to other.

Currently, use of apremilast and tofacitinib favors an early healing of nail psoriasis because they act directly on the pathogenic targets, distressing the inflammatory signals associated with the initiation and maintenance of the disease activity without altering the microbial flora and, as with several conventional synthetic DMARDs, they are characterized by the convenience of oral administration. ${ }^{44,84}$ The Medical Board of the National Psoriasis Foundation recommends systemic treatments, including apremilast, for patients with major skin and nail disease or skin, nail, and joint disease. ${ }^{44}$

\section{Conclusion}

This review underlines that the number of treatments for PsA and psoriasis has increased considerably in recent years 
and will probably lead to the authorization of new therapies in the future. Given the heterogeneity of the disease, the therapy should be personalized to individual cases. Principal features of these strategies should consider optimizing QOL, eliciting inclinations and goals, balancing risks and benefits, setting priorities, discontinuing needless medications, and starting beneficial medications, however, prioritizing simple regimens and integrating care. Models of care should be custom-made on patient necessity. Achieving this transition will require a change from disease-specific to patient-specific models of care delivery. ${ }^{88}$

Further investigations including prognostic biomarkers and patient stratification are necessary to allow clinicians the possibility of making better use of various biologic and non-biologic treatment options available.

\section{Disclosure}

The authors report no conflicts of interest in this work.

\section{References}

1. Parisi R, Symmons DP, Griffiths CE, Ashcroft DM; Identification and Management of Psoriasis and Associated ComorbidiTy (IMPACT) project team. Global epidemiology of psoriasis: a systematic review of incidence and prevalence. J Invest Dermatol. 2013;133(2):377-385.

2. Gordon KB, Strober BE. Understanding therapeutic pathways and comorbidities in psoriasis. Semin Cutan Med Surg. 2014;33(2 Suppl 2): S20-S23.

3. Davidovici BB, Sattar N, Prinz J, et al. Psoriasis and systemic inflammatory diseases: potential mechanistic links between skin disease and co-morbid conditions. J Invest Dermatol. 2010;130(7):1785-1796.

4. Gottlieb AB, Dann F. Comorbidities in patients with psoriasis. Am JMed. 2009;122(12):1150.e1-e9.

5. Gisondi P, Ferrazzi A, Girolomoni G. Metabolic comorbidities and psoriasis. Acta Dermatovenerol Croat. 2010;18(4):297-304.

6. Esposito M, Saraceno R, Giunta A, Maccarone M, Chimenti S. An Italian study on psoriasis and depression. Dermatology. 2006;212(2): 123-127.

7. Esposito M, Faleri S, Babino G, et al. From patients' needs to treatment outcomes in psoriasis: results from the 'pSORRIDI' experience. $J$ Int Med Res. 2016;44(1 Suppl 1):95-99.

8. Tham SN, Lim JJ, Tay Sh Chiew YF, Chua TN, Tan E, Tan T. Clinical observations on nail changes in psoriasis. Ann Acad Med Singapore. 1988;17(4):482-485

9. Baran R, de Berker DAR, Holzberg M, Thomas L, editors. Baran and Dawber's Diseases of the Nail and Their Management. 4th ed. WileyBlackwell; 2012.

10. Heydendael VM, De Borgie CA, Spuls PI, Bossuyt PM, Bos JD, De Rie MA. The burden of psoriasis is not determined by disease severity only. J Investig Dermatol Symp Proc. 2004;9:131-135.

11. Reich K. Approach to managing patients with nail psoriasis. $J$ Eur Acad Dermatol Venereol. 2009;23(Suppl 1):15-21.

12. Pasch MC. Nail psoriasis: a review of treatment options. Drugs. 2016; 76:675-705.

13. de Jong EM, Seegers BA, Gulinck MK, Boezeman JB, van de Kerkhof PC. Psoriasis of the nails associated with disability in a large number of patients: results of a recent interview with 1,728 patients. Dermatology. 1996;193(4):300-303.

14. Lawry M. Biological therapy and nail psoriasis. Dermatol Ther. 2007; 20(1):60-67.
15. Armesto $\mathrm{S}$, Esteve A, Coto-Segura $\mathrm{P}$, et al. Nail psoriasis in individuals with psoriasis vulgaris: a study of 661 patients. Actas Dermosifiliogr. 2011;102(5):365-372. Spanish.

16. McGonagle D, Ash Z, Dickie L, McDermott M, Aydin SZ. The early phase of psoriatic arthritis. Ann Rheum Dis. 2011;70(Suppl 1): i71-i76.

17. Langley RG, Dauden E. Treatment and management of psoriasis with nail involvement: a focus on biologic therapy. Dermatology. 2010; 221(Suppl 1):29-42.

18. Klaassen KM, van de Kerkhof PC, Pasch MC. Nail psoriasis: a questionnaire-based survey. Br J Dermatol. 2013;169(2):314-319.

19. Jiaravuthisan MM, Sasseville D, Vender RB, Murphy F, Muhn CY. Psoriasis of the nail: anatomy, pathology, clinical presentation, and a review of the literature on therapy. J Am Acad Dermatol. 2007;57(1):1-27.

20. Langenbruch A, Radtke MA, Krensel M, Jacobi A, Reich K, Augustin M. Nail involvement as a predictor of concomitant psoriatic arthritis in patients with psoriasis. Br J Dermatol. 2014;171(5):1123-1128.

21. Zaias N. Psoriasis of the nail. A clinical-pathologic study. Arch Dermatol. 1969;99(5):567-579.

22. Rich P, Scher RK. Nail psoriasis severity index: a useful tool for evaluation of nail psoriasis. J Am Acad Dermatol. 2003;49(2):206-212.

23. Szepietowski JC, Salomon J. Do fungi play a role in psoriatic nails? Mycoses. 2007;50(6):437-442.

24. Rigopoulos D, Papanagiotou V, Daniel R 3rd, Piraccini BM. Onychomycosis in patients with nail psoriasis: a point to point discussion. Mycoses. 2017;60(1):6-10.

25. Elewski BE. Onychomycosis: pathogenesis, diagnosis, and management. Clin Microbiol Rev. 1998;11(3):415-429.

26. Zaias N, Escovar SX, Zaiac MN. Finger and toenail onycholysis. J Eur Acad Dermatol Venereol. 2015;29(5):848-853.

27. Zisova L, Valtchev V, Sotiriou E, Gospodinov D, Mateev G. Onychomycosis in patients with psoriasis: a multicentre study. Mycoses. 2012; 55(2):143-147.

28. Nestle FO, Kaplan DH, Barker J. Psoriasis. N Engl J Med. 2009;361(5): 496-509.

29. Mak RK, Hundhausen C, Nestle FO. Progress in understanding the immunopathogenesis of psoriasis. Actas Dermosifiliogr. 2009; 100(Suppl 2):2-13.

30. De Luca A, Zelante T, D'Angelo C, et al. IL-22 defines a novel immune pathway of antifungal resistance. Mucosal Immunol. 2010;3(4): 361-373.

31. Kisand K, Bøe Wolff AS, Podkrajsek KT, et al. Chronic mucocutaneous candidiasis in APECED or thymoma patients correlates with autoimmunity to Th17-associated cytokines. J Exp Med. 2010;207(2): 299-308.

32. Huppler AR, Conti HR, Herna' ndez-Santos N, Darville T, Biswas PS, Gaffen SL. Role of neutrophils in IL-17-dependent immunity to mucosal candidiasis. J Immunol. 2014;192(4):1745-1752.

33. Kagami S, Rizzo HL, Kurtz SE, Miller LS, Blauvelt A. IL-23 and IL-17A, but not IL-12 and IL-22, are required for optimal skin host defense against Candida albicans. J Immunol. 2010;185(9):5453-5462.

34. Waldman A, Gilhar A, Duek L, Berdicevsky I. Incidence of Candida in psoriasis - a study on the fungal flora of psoriatic patients. Mycoses. 2001;44(3-4):77-81.

35. Taheri Sarvtin M, Shokohi T, Hajheydari Z, Yazdani J, Hedayati MT. Evaluation of candidal colonization and specific humoral responses against Candida albicans in patients with psoriasis. Int J Dermatol. 2014;53(12):e555-e560.

36. Fry L, Baker BS. Triggering psoriasis: the role of infections and medications. Clin Dermatol. 2007;25(6):606-615.

37. Goldminz AM, Au SC, Kim N, Gottlieb AB, Lizzul PF. NF-кB: an essential transcription factor in psoriasis. J Dermatol Sci. 2013; 69(2):89-94

38. Rashmi R, Rao KS, Basavaraj KH. A comprehensive review of biomarkers in psoriasis. Clin Exp Dermatol. 2009;34(6):658-663.

39. Di Meglio P, Perera GK, Nestle FO. The multitasking organ: recent insights into skin immune function. Immunity. 2011;35(6):857-869. 
40. Iyer SS, Cheng G. Role of interleukin 10 transcriptional regulation in inflammation and autoimmune disease. Crit Rev Immunol. 2012; 32(1):23-63.

41. Saulite I, Pilmane M, Kisis J. Expression of antimicrobial peptides in nail psoriasis and normal nails. Acta Derm Venereol. 2017;97(5): 644-645.

42. McGonagle D, Benjamin M, Tan AL. The pathogenesis of psoriatic arthritis and associated nail disease: not autoimmune after all? Curr Opin Rheumatol. 2009;21(4):340-347.

43. Gniadecki R. Next-generation antipsoriatic drugs: small molecules join. Br J Dermatol. 2015;173(6):1355-1356.

44. Crowley JJ, Weinberg JM, Wu JJ, Robertson AD, Van Voorhees AS; National Psoriasis Foundation. Treatment of nail psoriasis: best practice recommendations from the Medical Board of the National Psoriasis Foundation. JAMA Dermatol. 2015;151(1):87-94.

45. Saggini A, Chimenti S, Chiricozzi A. IL-6 as a druggable target in psoriasis: focus on pustular variants. J Immunol Res. 2014;2014:964069.

46. Manhart R, Rich P. Nail Psoriasis. Clin Exp Rheumatol. 2015; 33:S7-S13.

47. Marquez Balbas G, Sanchez Regana M, Umbert Millet P. Tacalcitol ointment for the treatment of nail psoriasis. J Dermatolog Treat. 2009; 20(5):308-310.

48. Reichrath J. Vitamin D and the skin: an ancient friend, revisited. Exp Dermatol. 2007;16(7):618-625.

49. Scher RK, Stiller M, Zhu YI. Tazarotene $0.1 \%$ gel in the treatment of fingernail psoriasis: a double-blind, randomized, vehicle-controlled study. Cutis. 2001;68(5):355-358.

50. Bianchi L, Soda R, Diluvio L, Chimenti S. Tazarotene $0.1 \%$ gel for psoriasis of the fingernails and toenails: an open, prospective study. Br J Dermatol. 2003;149(1):207-209.

51. Campione E, Paternò EJ, Costanza G, et al. Tazarotene as alternative topical treatment for onychomycosis. Drug Des Devel Ther. 2015;9: 879-886.

52. Soda R, Diluvio L, Bianchi L, Chimenti S. Treatment of trachyonychia with tazarotene. Clin Exp Dermatol. 2005;30(3):301-302.

53. Fischer-Levancini C, Sanchez-Regana M, Llambi F, Collgros H, Exposito-Serrano V, Umbert-Millet P. Nail psoriasis: treatment with tazarotene 0.1\% hydrophilic ointment. Actas Dermosifiliogr. 2012; 103(8):725-728. Spanish.

54. Blauvelt A, Armstrong AW, Krueger GG. Essential truths for the care and management of moderate-to-severe psoriasis. J Drugs Dermatol. 2015;14(8):805-812.

55. Gumusel M, Ozdemir M, Mevlitoglu I, Bodur S. Evaluation of the efficacy of methotrexate and cyclosporine therapies on psoriatic nails: a one-blind, randomized study. J Eur Acad Dermatol Venereol. 2011;25(9):1080-1084.

56. Reich K, Langley RG, Papp KA, et al. A 52-week trial comparing briakinumab with methotrexate in patients with psoriasis. $N$ Engl $J$ Med. 2011;365(17):1586-1596.

57. Sanchez-Regana M, Sola-Ortigosa J, Alsina-Gibert M, VidalFernandez M, Umbert-Millet P. Nail psoriasis: a retrospective study on the effectiveness of systemic treatments (classical and biological therapy). J Eur Acad Dermatol Venereol. 2011;25(5):579-586.

58. Demirsoy EO, Kiran R, Salman S, et al. Effectiveness of systemic treatment agents on psoriatic nails: a comparative study. J Drugs Dermatol. 2013;12(9):1039-1043.

59. Syuto T, Abe M, Ishibuchi H, Ishikawa O. Successful treatment of psoriatic nails with low-dose cyclosporine administration. Eur J Dermatol. 2007;17(3):248-249.

60. Karanikolas GN, Koukli EM, Katsalira A, et al. Adalimumab or cyclosporine as monotherapy and in combination in severe psoriatic arthritis: results from a prospective 12-month nonrandomized unblinded clinical trial. J Rheumatol. 2011;38(11):2466-2474.

61. Ricceri F, Pescitelli L, Tripo L, Bassi A, Prignano F. Treatment of severe nail psoriasis with acitretin: an impressive therapeutic result. Dermatol Ther. 2013;26(1):77-78.
62. Tosti A, Ricotti C, Romanelli P, Cameli N, Piraccini BM. Evaluation of the efficacy of acitretin therapy for nail psoriasis. Arch Dermatol. 2009;145(3):269-271.

63. Lowes MA, Suarez-Farinas M, Krueger JG. Immunology of psoriasis. Annu Rev Immunol. 2014;32:227-255.

64. Pirowska MM, Goździalska A, Lipko-Godlewska S, et al. Autoimmunogenicity during anti-TNF therapy in patients with psoriasis and psoriatic arthritis. Postepy Dermatol Alergol. 2015;32(4): 250-254.

65. Bianchi L, Bergamin A, de Felice C, Capriotti E, Chimenti S. Remission and time of resolution of nail psoriasis during infliximab therapy. $J$ Am Acad Dermatol. 2005;52(4):736-737.

66. Rigopoulos D, Gregoriou S, Lazaridou E, et al. Treatment of nail psoriasis with adalimumab: an open label unblinded study. J Eur Acad Dermatol Venereol. 2010;24(5):530-534.

67. Barrera MV, Habicheyn S, Mendiola MV, Herrera Ceballos E. Etanercept in the treatment and retreatment of psoriasis in daily clinical practice. Eur J Dermatol. 2008;18(6):683-687.

68. Rallis E, Stavropoulou E, Rigopoulos D, Verros C. Rapid response of nail psoriasis to etanercept. J Rheumatol. 2008;35(3):544-545.

69. Coelho JD, Diamantino F, Lestre S, Ferreira AM. Treatment of severe nail psoriasis with etanercept. Indian J Dermatol Venereol Leprol. 2011;77(1):72-74.

70. Kavanaugh A, van der Heijde D, McInnes IB, et al. Golimumab in psoriatic arthritis: one-year clinical efficacy, radiographic, and safety results from a phase III, randomized, placebo-controlled trial. Arthritis Rheum. 2012;64(8):2504-2517.

71. Mease PJ, Fleischmann R, Deodhar AA, et al. Effect of certolizumab pegol on signs and symptoms in patients with psoriatic arthritis: 24-week results of a Phase 3 double-blind randomised placebo-controlled study (RAPID-PsA). Ann Rheum Dis. 2014;73(1):48-55.

72. Park H, Li Z, Yang XO, et al. A distinct lineage of CD4 T cells regulates tissue inflammation by producing interleukin 17. Nat Immunol. 2005;6(11):1133-1141.

73. Cai Y, Fleming C, Yan J. Dermal $\gamma \delta \mathrm{T}$ cells - a new player in the pathogenesis of psoriasis. Int Immunopharmacol. 2013;16(3):388-391.

74. Rich P, Bourcier M, Sofen H, et al; PHOENIX 1 investigators. Ustekinumab improves nail disease in patients with moderate-to-severe psoriasis: results from PHOENIX 1. Br J Dermatol. 2014;170(2): 398-407.

75. Rigopoulos D, Gregoriou S, Makris M, Ioannides D. Efficacy of ustekinumab in nail psoriasis and improvement in nail-associated quality of life in a population treated with ustekinumab for cutaneous psoriasis: an open prospective unblinded study. Dermatology. 2011;223(4): 325-329.

76. Patsatsi A, Kyriakou A, Sotiriadis D. Ustekinumab in nail psoriasis: an open-label, uncontrolled, nonrandomized study. J Dermatol Treat. 2013;24(2):96-100

77. Igarashi A, Kato T, Kato M, Song M, Nakagawa H; Japanese Ustekinumab Study Group. Efficacy and safety of ustekinumab in Japanese patients with moderate-to severe plaque-type psoriasis: long-term results from a phase 2/3 clinical trial. J Dermatol. 2012;39(3): 242-252.

78. Yao Z, Painter SL, Fanslow WC, et al. Human IL-17: a novel cytokine derived from T cells. J Immunol. 1995;155(12):5483-5486.

79. Gaffen SL, Jain R, Garg AV, Cua DJ. The IL-23-IL-17 immune axis: from mechanisms to therapeutic testing. Nat Rev Immunol. 2014;14(9): 585-600.

80. Gaffen SL. The role of interleukin-17 in the pathogenesis of rheumatoid arthritis. Curr Rheumatol Rep. 2009;11(5):365-370.

81. Leonardi C, Matheson R, Zachariae C, et al. Anti-interleukin-17 monoclonal antibody ixekizumab in chronic plaque psoriasis. $N$ Engl J Med. 2012;366(13):1190-1199.

82. Langley RG, Rich P, Menter A, et al. Improvement of scalp and nail lesions with ixekizumab in a phase 2 trial in patients with chronic plaque psoriasis. J Eur Acad Dermatol Venereol. 2015;29(9):1763-1770. 
83. Thaçi D, Kimball A, Foley P, et al. Apremilast, an oral phosphodiesterase 4 inhibitor, improves patient-reported outcomes in the treatment of moderate to severe psoriasis: results of two phase III randomized, controlled trials. J Eur Acad Dermatol Venereol. 2016;31(3): 498-506.

84. Papp K, Reich K, Leonardi CL, et al. Apremilast, an oral phosphodiesterase 4 (PDE4) inhibitor, in patients with moderate to severe plaque psoriasis: results of a phase III, randomized, controlled trial (Efficacy and Safety Trial Evaluating the Effects of Apremilast in Psoriasis [ESTEEM] 1). J Am Acad Dermatol. 2015;73(1):37-49.

85. Rich P, Gooderham M, Bachelez H, et al. Apremilast, an oral phosphodiesterase 4 inhibitor, in patients with difficult-to-treat nail and scalp psoriasis: results of 2 phase III randomized, controlled trials (ESTEEM 1 and ESTEEM 2). J Am Acad Dermatol. 2016;74(1):134-142.
86. Papp KA, Menter MA, Abe M, et al; OPT Pivotal 1 and OPT Pivotal 2 investigators. Tofacitinib, an oral Janus kinase inhibitor, for the treatment of chronic plaque psoriasis: results from two randomized, placebocontrolled, phase III trials. Br J Dermatol. 2015;173(4):949-961.

87. Fénix-Caballero S, Alegre-del Rey EJ, Castaño-Lara R, PuigventósLatorre F, Borrero-Rubio JM, López-Vallejo JF. Direct and indirect comparison of the efficacy and safety of adalimumab, etanercept, infliximab and golimumab in psoriatic arthritis. J Clin Pharm Ther. 2013;38(4):286-293.

88. Thorlund K, Druyts E, Aviña-Zubieta JA, Mills EJ. Anti-tumor necrosis factor (TNF) drugs for the treatment of psoriatic arthritis: an indirect comparison meta-analysis. Biologics. 2012;6:417-427.

89. Bierman AS, Tinetti ME. Precision medicine to precision care: managing multimorbidity. Lancet. 2016;388(10061):2721-2723.

\section{Publish your work in this journal}

Drug Design, Development and Therapy is an international, peerreviewed open-access journal that spans the spectrum of drug design and development through to clinical applications. Clinical outcomes, patient safety, and programs for the development and effective, safe, and sustained use of medicines are the features of the journal, which has also been accepted for indexing on PubMed Central. The manuscript management system is completely online and includes a very quick and fair peer-review system, which is all easy to use. Visit http://www.dovepress.com/testimonials.php to read real quotes from published authors.

Submit your manuscript here: http://www.dovepress.com/drug-design-development-and-therapy-journal 